\title{
Avaliação de substratos de oviposição para Orius insidiosus (Say) (Hemiptera, Anthocoridae)
}

\author{
Lívia M. Carvalho ${ }^{1}$, Vanda H. P. Bueno² \& Cristina Castañé ${ }^{3}$
}

${ }^{1}$ Empresa de Pesquisa Agropecuária de Minas Gerais (EPAMIG), Fazenda Experimental Risoleta Neves (FERN), Av. Visconde do Rio Preto, s/n, Vila São Paulo (Fábricas). 36301-360 São João Del Rei-MG, Brasil. livia@epamig.br

${ }^{2}$ Departamento de Entomologia, Universidade Federal de Lavras (UFLA), Caixa Postal 3037, 37200-000 Lavras-MG, Brasil. vhpbueno@ufla.br

${ }^{3}$ Departamento de Proteção Vegetal, Institute de Recerca e Tecnologia Agroalimentarie (IRTA), Centre de Cabrils, Ctra. Cabrils s/n, 08348 Cabrils (Barcelona), Espanha. cristina.castane@irta.es

\begin{abstract}
Evaluation of oviposition substrates for Orius insidiosus (Say) (Hemiptera, Anthocoridae). Females of $O$. insidiosus deposit their eggs in the plant tissue. This study aimed to evaluate oviposition substrates for this predator. The study was conducted in an air-conditioned room at $25 \pm 2{ }^{\circ} \mathrm{C}, 70 \pm 10 \% \mathrm{RH}$, and a $12 \mathrm{~h}$ photophase. The oviposition substrates used consisted of bean sprouts (Phaseolus vulgaris L.), soybean sprouts [Glycine max (L.) Merr.], potato sprouts (Solanum tuberosum L.), bean pods (Phaseolus vulgaris L.), and farmer's friend inflorescences (Bidens pilosa L.). Evaluations included the daily mean and total numbers of eggs per female during a 15-day period, the number of live adults in each container, and adult production viability. All substrates tested were accepted by the females. However, a significantly higher number of $O$. insidiosus eggs was found on bean sprouts (4.3 eggs per day) and soybean sprouts (3.9 eggs per day), when compared with the other substrates evaluated. The lowest $(\mathrm{p}<0,05)$ viability values in the production of $O$. insidiosus adults $(75.1$ and $71.7 \%)$ were observed from eggs laid on potato sprouts and bean pods, respectively. Bean and soybean sprouts were suitable to rear $O$. insidiosus in the laboratory; these substrates have the additional advantages of being produced throughout the year without requiring large areas for its production, thus reducing the costs and labor necessary to obtain and prepare them to be use on the mass-rearing system. These results may be useful in the mass rearing of $O$. insidiosus in the laboratory, with the objective of releasing the predator in biological control programs against thrips.
\end{abstract}

KEYWORDS. Biology; biological control; fecundity; predator.

RESUMO. Avaliação de substratos de oviposição para Orius insidiosus (Say) (Hemiptera, Anthocoridae). Fêmeas do predador $O$. insidiosus usam tecidos de plantas para colocação de seus ovos, o que caracteriza a oviposição endofítica. Este trabalho teve por objetivo avaliar diferentes substratos de oviposição para este predador. O estudo foi conduzido em sala climatizada a $25 \pm 2^{\circ} \mathrm{C}, \mathrm{UR}$ de $70 \pm 10 \%$ e fotofase de 12 horas. Os substratos de oviposição utilizados foram brotos de feijão (Phaselus vulgaris L.), brotos de soja [Glycine max (L.) Merr.], brotos de batata (Solanum tuberosum L.), vagem de feijão (Phaselus vulgaris L.) e inflorescências de picão-preto (Bidens pilosa L.). Foram avaliados os números médio diário e total de ovos por um período de 15 dias, o número de adultos vivos em cada recipiente e a viabilidade na produção dos adultos. Todos os substratos testados foram aceitos pelas fêmeas. Entretanto, foi observado um número significativamente maior de ovos de $O$. insidiosus em brotos de feijão (4,3 ovos por dia) e brotos de soja ( 3,9 ovos por dia), comparado aos demais substratos avaliados. As menores $(p<0,05)$ viabilidades na produção de adultos de $O$. insidiosus $(75,1$ e $71,7 \%$ ) foram verificadas a partir dos ovos colocados em brotos de batata e vagem de feijão, respectivamente. Brotos de feijão e brotos de soja foram adequados para a utilização na criação de $O$. insidiosus em laboratório, com vantagens de poderem ser produzidos durante todo o ano sem necessitar de grandes áreas para isso, reduzindo assim os custos e o trabalho com a sua obtenção e preparo para uso no sistema de criação do predador. Esses resultados poderão auxiliar na criação massal de $O$. insidiosus em laboratório, visando à liberação do mesmo em programas de controle biológico de tripes.

PALAVRAS-CHAVE. Biologia; controle biológico; fecundidade; predador.

A oviposição endofítica em plantas é uma característica observada entre as espécies de Orius Wolff, 1811 (Bueno 2000) e vários substratos naturais e artificiais têm sido pesquisados para a criação desses agentes de controle (Castañé \& Zalom 1994; Richards \& Schmidt 1996). Os aspectos estruturais, nutricionais e químicos de diferentes plantas podem potencialmente influenciar o desempenho das fêmeas de Orius insidiosus (Say, 1832) (Hemiptera: Anthocoridae) sobre as plantas (Lundgren \& Fergen 2006). Assim, as plantas exercem grande importância na criação massal de predadores heterópteros, pois além de servirem de meio de oviposição, também são fonte de nutrientes e umidade (Coll 1998). Dessa maneira, um dos principais obstáculos para a criação massal de espécies de Orius é a escolha de um substrato de oviposição que seja aceito pela fêmea, de baixo custo e de fácil obtenção (Richards \& Schmidt 1996).

O substrato de oviposição comumente utilizado para criação de espécies de Orius é a vagem de feijão (Phaseolus vulgaris L.) (Isenhour \& Yeargan 1981; van den Meiracker 1999; Ruberson et al. 2000; Yano et al. 2002; Tommasini et al. 2004). No entanto, este substrato de oviposição muitas vezes apresenta contaminação por fungos e se decompõe antes dos ovos do predador terem completado seu desenvolvimento (Richards \& Schmidt 1996). Outros substratos como hastes de feijão, folhas de algodão e pepino (Bueno 2000), folhas de feijão (Honda et al. 1998), folhas de berinjela (Nagai \& 
Yano 1999) e inflorescências de picão-preto Bidens pilosa L. (Mendes et al. 2005) têm sido testados. Entretanto, também foi verificado que esses materiais requerem considerável espaço para a sua produção ou são difíceis de produzir (picão-preto), o que aumenta o custo de produção do agente biológico, além de possibilitarem a introdução de patógenos e/ou resíduos de inseticidas na criação. Pesquisas realizadas com brotos (sementes recém-geminadas) (Richards \& Schmidt 1996; Wang et al. 1999) têm evidenciado que estes apresentam baixo custo de produção, além de menor risco de introdução de patógenos nas criações (Murai et al. 2001).

Este trabalho teve por objetivo avaliar diferentes substratos de oviposição para a criação de $O$. insidiosus em laboratório e verificar a viabilidade destes na produção dos adultos, visando à maximização da criação do agente de controle biológico.

\section{MATERIAL E MÉTODOS}

O estudo foi conduzido no Laboratório de Controle Biológico do Departamento de Entomologia (UFLA), em sala climatizada a $25 \pm 11^{\circ} \mathrm{C}$, UR de $70 \pm 10 \%$ e fotofase de 12 horas. Os insetos utilizados foram provenientes da criação de manutenção do laboratório, onde permaneceram até a fase adulta em placas de Petri de $20 \mathrm{~cm}$, contendo local de abrigo (papel toalha dobrado), fonte de água (algodão umedecido) e alimento ad libitum [ovos de Anagasta kuehniella (Zeller, 1879) (Lepidoptera, Pyralidae)], de acordo com metodologia proposta por Bueno (2000) e Bueno et al. (2007).

Obtenção e formas de utilização dos substratos de oviposição. Como substratos de oviposição para $O$. insidiosus foram utilizados brotos de feijão (Phaselus vulgaris L.), brotos de soja [Glycine $\max$ (L.) Merr.], inflorescências de picão-preto (Bidens pilosa L.), vagens de feijão e brotos de batata (Solanum tuberosum L.).

Os brotos de feijão e de soja foram obtidos por meio do plantio de sementes em bandejas plásticas de $20 \times 40 \times 10 \mathrm{~cm}$, contendo algodão umedecido no fundo e papel filtro úmido sobre as sementes visando evitar a sua dessecação. Estas bandejas foram mantidas no escuro em câmara climatizada na temperatura de $25 \pm 1^{\circ} \mathrm{C}$ e UR de $70 \pm 10 \%$ por três a quatro dias para germinação das sementes (estádio de cotilédone). Posteriormente, foi retirado o tegumento (casca) da semente germinada e preparado um bouquet com seis brotos de aproximadamente $10 \mathrm{~cm}$ de comprimento cada, envolvidos em chumaço de algodão.

As inflorescências de picão-preto foram coletadas no campo e avaliadas sob microscópio estereoscópico para garantir que não houvessem posturas prévias no substrato. Posteriormente, foi preparado bouquet utilizando cinco a seis hastes (10 cm cada). As vagens de feijão utilizadas nos testes foram adquiridas no mercado local e colocadas de cinco a seis vagens de feijão, com aproximadamente $10 \mathrm{~cm}$ cada. Ambos os substratos foram tratados anteriormente em solução de hipoclorito de sódio a $0,12 \%$, por um período de quatro minutos para a sua desinfecção de acordo com metodologia proposta por Diniz et al. (2006).

Os brotos de batata foram obtidos através do uso de batata- semente, evitando-se o uso de tubérculos infectados com viroses e bacterioses. A batata-semente apresenta dormência e as gemas permanecem inaptas à brotação durante certo período. Para ativação das brotações foi utilizado bissulfureto de carbono conforme metodologia proposta por Silva et al. (2004). A batata utilizada como substrato de oviposição apresentava cinco a seis brotações de aproximadamente 10 $\mathrm{cm}$.

Avaliação da fecundidade de $O$. insidiosus nos diferentes substratos de oviposição. Cada tratamento consistiu de um recipiente de vidro de 1,7 litros contendo ovos de $A$. kuehniella como alimento e papel toalha dobrado como abrigo, onde foram acrescentados 25 casais de $O$. insidiosus com até 48 horas de vida, obtidos da criação de manutenção do laboratório, e um tipo de substrato de oviposição. Como fonte de umidade foi colocado em cada recipiente água em vidro $(10 \mathrm{ml})$ contendo algodão na parte superior. Para cada tratamento foram realizadas quatro repetições.

Os substratos de oviposição foram trocados a cada dois dias, ocasião na qual também foram adicionados alimento e água. Cada substrato de oviposição retirado foi examinado sob microscópio estereoscópico para contagem do número de ovos, avaliando-se o número médio diário e total de ovos/ fêmea por um período de 15 dias. Esse período foi adotado em função da maior parte dos ovos produzidos pelas espécies de Orius serem colocados nos primeiros 15 dias após a sua emergência como foi comprovado para $O$. insidiosus (Castañé \& Zalom 1994; Bueno et al. 2006), Orius laevigatus (Fieber, 1860) (Alauzet et al. 1994; Blumel 1996; Tommasini et al. 2004), Orius majusculus (Reuter, 1879) (Blumel 1996) e Orius thyestes Herring, 1966 (Carvalho et al. 2005).

Após a contagem do número de ovos, os substratos de oviposição foram transferidos para placas de Petri de $20 \mathrm{~cm}$. Dentro de cada placa foi acrescentado papel toalha dobrado e, a cada dois dias, foram colocados ovos de A. kuehniella como alimento para o desenvolvimento das ninfas e algodão umedecido, até essas se tornarem adultos. Todos os substratos de oviposição foram mantidos nas placas junto das ninfas até a emergência dos adultos, fornecendo umidade e abrigo para as ninfas, evitando o canibalismo. Após a emergência dos adultos foi calculada a percentagem de ovos que deram origem a adultos, ou seja, a viabilidade de ovo a adulto em cada substrato de oviposição, utilizando-se a fórmula: viabilidade ovo-adulto $\%=\left(\mathrm{n}^{\mathrm{o}}\right.$ de adultos obtidos. 100$) / \mathrm{n}^{\circ}$ de ovos.

Análise dos dados. Para avaliar o número de ovos por fêmea por dia, o número total de ovos por fêmea e a viabilidade de ovo a adulto, procedeu-se a análise de variância e a média dos tratamentos foi comparada pelo teste de Scott-Knott com um nível nominal de significância de 5\%.

\section{RESULTADOS E DISCUSSÃO}

Fêmeas de $O$. insidiosus colocaram seus ovos nos diferentes substratos de oviposição avaliados, embora tenha sido observada preferência por alguns substratos testados. 
Também foi observada preferência por locais para oviposição nos substratos avaliados. Nos brotos de feijão e de soja, os ovos geralmente foram colocados próximo ao cotilédone; nas inflorescências de picão-preto os ovos foram colocados na base da haste de cada inflorescência. Na vagem de feijão as fêmeas de $O$. insidiosus inseriram os ovos nas nervuras longitudinais e nos brotos de batata os ovos foram colocados na base das brotações. O comportamento de oviposição endofitica é uma característica observada entre os predadores do gênero Orius (Bueno 2000) que permite uma maior proteção dos ovos tanto contra os possíveis inimigos naturais e também contra os fatores adversos, como o dessecamento, assegurando assim o seu desenvolvimento. Richards \& Schmidt (1996) relataram que as fêmeas de $O$. insidiosus selecionaram para a oviposição locais protegidos e que não estão sujeitos a rápida dessecação. Também, Murai et al. (2001) verificaram que Orius sauteri (Poppius, 1909) colocaram os ovos em brotos de feijão somente próximos à base do cotilédone.

Fecundidade de Orius insidiosus. O desempenho reprodutivo de $O$. insidiosus foi diferente nos diversos substratos de oviposição testados. O número diário de ovos colocados por fêmeas foi significativamente maior quando $O$. insidiosus foi mantido com brotos de feijão ou brotos de soja (4,3 $\pm 0,28$ e $3,9 \pm 0,21$ ovos por dia, respectivamente), comparado aos demais substratos $(\mathrm{P}<0,05)$, resultando em um número total de ovos igual a $63,9 \pm 1,66$ e 59,7 $\pm 1,12$ ovos, respectivamente (Tabela I). Este fato pode estar relacionado com a relativa maciez e turgidez destes brotos, o que facilitou a oviposição, pois o teor de umidade pode ser um importante fator de estímulo para oviposição de $O$. insidiosus. De acordo com Lundgren \& Fergen (2006), a preferência de oviposição de $O$. insidiosus é influenciada por características físicas e morfológicas das plantas e a facilidade com que as fêmeas colocam seus ovos dentro do substrato afeta a aceitabilidade de algumas espécies de plantas. Segundo van den Meiracker \& Sabelis (1993), características do substrato como maciez e umidade influenciam a oviposição de $O$. insidiosus. Mendes et al. (2005) relataram que a preferência por substratos para oviposição de $O$. insidiosus pode envolver a facilidade de inserção do ovipositor e dos ovos e, de acordo com Richards \& Schmidt (1996), materiais com umidade suficiente para favorecer o desenvolvimento dos ovos de $O$. insidiosus foram mais aceitos que substratos mais secos.

Inflorescências de picão-preto se mostraram como substrato intermediário, ou seja, foram piores que os brotos de feijão e soja, mas melhores que a vagem de feijão e brotos de batata (Tabela I). Bueno et al. (2007) relataram que $O$. insidiosus colocaram 1,2 ovos por fêmea por dia em inflorescências de picão-preto, quando foram mantidos na densidade de 400 adultos por recipiente de 1,7 L. A diferença entre os resultados indica que em uma criação massal deste predador, o desempenho reprodutivo da fêmea pode cair devido ao efeito de agrupamento dos indivíduos dentro do recipiente de criação. Em altas densidades de $O$. insidiosus por recipiente pode ocorrer canibalismo e interferência mútua dos indivíduos reduzindo o padrão de oviposição das fêmeas,
Tabela I. Número médio diário e total de ovos $( \pm \mathrm{EP})$ de Orius insidiosus mantidos em diferentes substratos de oviposição $\left(25 \pm 2^{\circ} \mathrm{C}\right.$, UR de $70 \pm 10$ $\%$ e fotofase de $12 \mathrm{~h}$ ).

\begin{tabular}{ccc}
\hline $\begin{array}{c}\text { Substratos de } \\
\text { oviposição }\end{array}$ & $\begin{array}{c}\text { Número ovos/ } \\
\text { fêmea/dia }\end{array}$ & $\begin{array}{c}\text { Número ovos/ } \\
\text { fêmea }\end{array}$ \\
\hline Broto de feijão & $4,3 \pm 0,28 \mathrm{a}^{*}$ & $63,9 \pm 1,66 \mathrm{a}$ \\
Broto de soja & $3,9 \pm 0,22 \mathrm{a}$ & $59,7 \pm 1,12 \mathrm{a}$ \\
Picão-preto & $2,9 \pm 0,22 \mathrm{~b}$ & $43,1 \pm 1,57 \mathrm{~b}$ \\
Vagem de feijão & $1,2 \pm 0,11 \mathrm{c}$ & $18,5 \pm 0,39 \mathrm{c}$ \\
Broto de batata & $1,4 \pm 0,13 \mathrm{c}$ & $21,4 \pm 0,35 \mathrm{c}$ \\
\hline
\end{tabular}

*Médias seguidas de mesma letra na coluna, não difere entre si pelo teste de Scott e Knott, a $5 \%$ de probabilidade.

pois menor quantidade de alimento ingerida é alocada para a oviposição (van den Meiracker 1999).

O menor número de ovos de $O$. insidiosus foi observado quando foram utilizados vagem de feijão ou brotos de batata como substrato de oviposição (Tabela I). Mendes et al. (2005) também verificaram que $O$. insidiosus colocou o menor número de ovos em vagem de feijão, tanto em teste sem chance de escolha (5,2 ovos/fêmea), como em teste com chance de escolha ( 0,4 ovos/fêmea), entre a vagem de feijão, caules de caruru e feijão e inflorescência de picão-preto.

Acompanhando o padrão de oviposição das fêmeas de $O$. insidiosus em cada substrato avaliado, os números acumulados de ovos foram de 1.599, 1.513, 1.095, 536 e 462 ovos nos substratos broto de feijão, broto de soja, picão-preto, broto de batata e vagem de feijão, respectivamente (Fig. 1). Baseado em resultados de Bueno et al. (2006), onde foi medida a oviposição ao longo do tempo pode-se inferir que em um período de 30 dias, uma fêmea de $O$. insidiosus produzirá 22,7 ovos tendo picão como substrato de oviposição. Bueno et al. (2007) constataram uma produção total de 8.295 ovos de

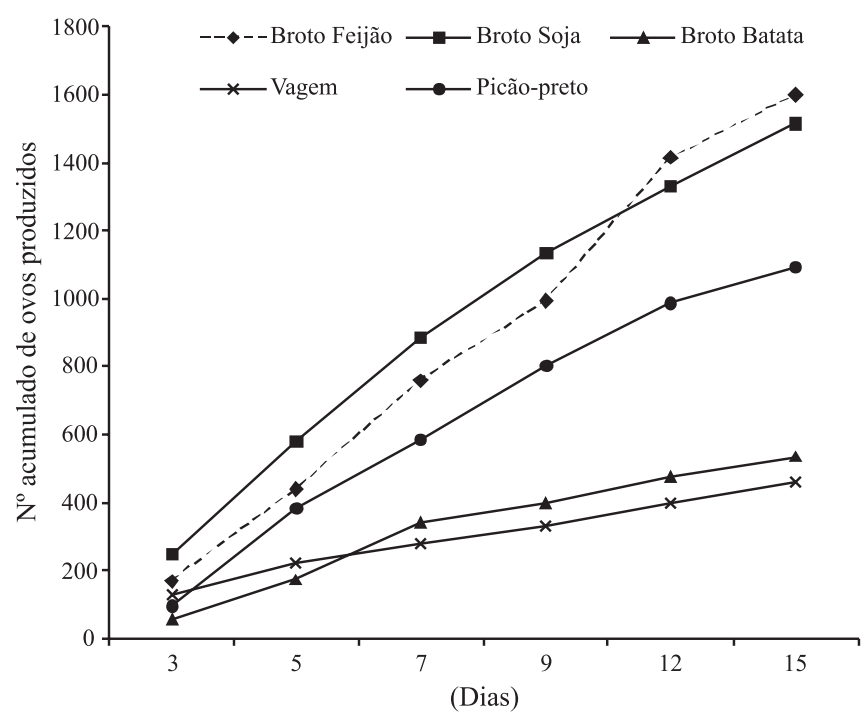

Fig. 1. Número acumulado de ovos produzidos por recipiente de criação de Orius insidiosus mantidos em diferentes substratos de oviposição $\left(25 \pm 2^{\circ} \mathrm{C}\right.$, UR de $70 \pm 10 \%$ e fotofase de $12 \mathrm{~h}$ ). 
O. insidiosus em inflorescência de picão-preto, por recipiente contendo 400 adultos no período de 30 dias.

Viabilidade na produção de adultos de Orius insidiosus. A viabilidade na produção de adultos de $O$. insidiosus foi significativamente diferente $(\mathrm{P}<0,05)$ nos diversos substratos de oviposição avaliados. As maiores viabilidades na produção de adultos de $O$. insidiosus foram observadas quando brotos de feijão $(87,8 \%)$, brotos de soja $(86,4 \%)$ ou inflorescências de picão-preto $(83,4 \%)$ foram usados como substrato de oviposição (Fig. 2). Wang et al. (1999) utilizaram brotos de feijão como substrato de oviposição e de fonte de água para adultos e ninfas de Orius strigicollis (Poppius, 1909) e relataram que as ninfas apresentaram um melhor desenvolvimento e sobrevivência sobre esse substrato, comparado com ninfas criadas sobre vagem de feijão ou folhas de berinjela. Murai et al. (2001) verificaram uma viabilidade de ovo a adulto de $90,3 \%$ para $O$. sauteri mantidos em brotos de feijão.

Apesar de vários estudos indicarem as inflorescências de picão-preto como substrato adequado para criação de O. insidiosus (Bueno 2000; Mendes et al. 2005; Bueno et al. 2006), foi verificado neste estudo que brotos de feijão e brotos de soja apresentaram melhores resultados quanto à produção de ovos e de adultos de $O$. insidiosus, sendo assim considerados mais adequados frente aos outros substratos avaliados (Fig. 1). Além disso, verifica-se que os brotos são mais fáceis de serem obtidos, uma vez que inflorescências de picão-preto são difíceis de serem cultivadas e sua obtenção fica dependente da realização de coletas no campo, ou seja, sujeitas às variações bióticas e abióticas. Uma opção poderia ser o cultivo de picão-preto, mas Carmona \& Villas Boas (2001) verificaram problemas na germinação de sementes de picão principalmente devido à perda de viabilidade causada por deterioração ou dormência das sementes.

Vagem de feijão e brotos de batata apresentaram as menores viabilidades quanto á produção de adultos de $O$. insidiosus, com 75,5 e 71,7\%, respectivamente, comparadas aos demais substratos (Fig. 2). Para a vagem de feijão, a menor viabilidade na produção de adultos pode ter ocorrido devido à contaminação por patógenos, os quais ocasionaram a decomposição do substrato mesmo antes da eclosão das ninfas, pois a desinfecção com hipoclorito não foi eficiente; além disso, foi observado o dessecamento desse material. Richards $\&$ Schmidt (1996) observaram que o pré-tratamento da vagem de feijão com solução de hipoclorito de sódio não preveniu o crescimento de fungos e quando as ninfas de primeiro instar de $O$. insidiosus eclodiam, ficavam aderidas aos micélios dos fungos e assim, eram incapazes de se alimentarem. Castañé \& Zalom (1994) acrescentam que o uso de vagens de feijão como substrato de oviposição pode ser problemático devido á sua limitada disponibilidade ao longo do ano, além do potencial de introdução de patógenos e resíduos de inseticidas dentro da criação. Outro problema é que quando se produz vagem de feijão sem inseticidas, o plantio deve ser feito em ambiente totalmente protegido, pois se cultivada no campo irá produzir vagens que poderão introduzir na criação outros

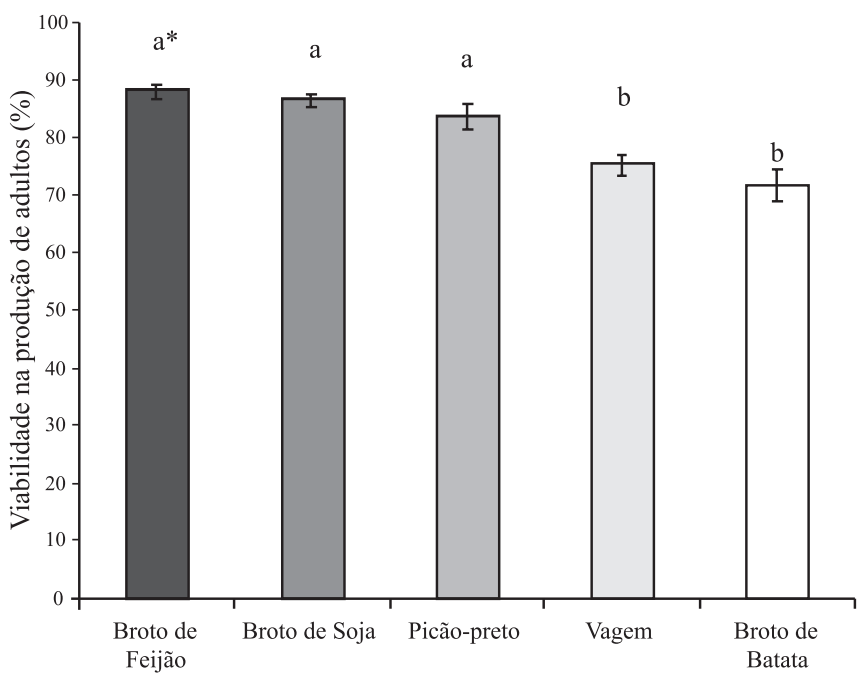

Fig. 2. Viabilidade (\%) ( \pm EP) na produção de adultos de Orius insidiosus criados em diferentes substratos de oviposição $\left(25 \pm 2^{\circ} \mathrm{C}\right.$, UR de $70 \pm 10 \%$ e fotofase de $12 \mathrm{~h}$ ).

*Médias com mesma letra não diferem entre si pelo teste Scott e Knott, com um nível nominal de significância de 5\%.

predadores que ovipositam muito bem em vagem de feijão no campo, como Franklinothrips vespiformis (Crawford, 1909) (Thysanoptera: Aeolothripidae). Sua postura é endofítica e difícil de ser observada ao microscópio estereoscópico, sendo este um problema sério para uso desse substrato.

Também o baixo número de ovos produzidos e a reduzida viabilidade na produção de adultos de $O$. insidiosus observados nos brotos de batata como substrato de oviposição podem ter sido ocasionados pela presença de resíduos químicos. De acordo com Filgueira (2000) e Pereira \& Fortes (2004), um dos fatores limitantes na produção de batata é sua suscetibilidade a grande número de doenças e pragas, sendo necessário o uso de produtos químicos para obtenção de tubérculos de qualidade.

Os resultados obtidos evidenciam que brotos de feijão ou brotos de soja foram adequados à criação de $O$. insidiosus, tanto maximizando a produção de ovos do predador mantido coletivamente (50 adultos por recipiente), como viabilizando a produção de adultos obtidos a partir desses ovos. Além disso, esses substratos foram fáceis de serem preparados e apresentaram a vantagem de serem produzidos com a mesma qualidade durante todo o ano. De acordo com Richards \& Schmidt (1996), os fatores que influenciam a adoção de um substrato de oviposição são a aceitação das fêmeas para oviposição, a sobrevivência dos ovos e desenvolvimento de ninfas no substrato, além do custo e possibilidade de incorporação desse substrato num programa de criação em larga escala.

Esses resultados poderão auxiliar na criação massal de $O$. insidiosus em laboratório. Contudo, estudos posteriores são necessários com a utilização de brotos de feijão e soja como substratos de oviposição em recipientes de criação contendo maior densidade de adultos de $O$. insidiosus, verificando se os índices de oviposição e viabilidade aqui obtidos se mantém, o que poderia maximizar a produção massal desse agente 
de controle para a sua liberação em sistemas de cultivos protegidos para o controle de tripes-praga.

Agradecimentos. Ao Conselho Nacional de Desenvolvimento Científico e Tecnológico (CNPq) pela bolsa de estudo e a Fundação de Amparo a Pesquisa do Estado de Minas Gerais (FAPEMIG) pelo apoio financeiro.

\section{REFERÊNCIAS}

Alauzet, A.; D. Dargagnon \& J. C. Malausa. 1994. Bionomics of a polyphagous predator: Orius laevigatus (Het.: Anthocoridae). Entomophaga 39: 33 40.

Blumel, S. 1996. Effect of selected mass-rearing parameters on Orius majusculus (Reuter) and Orius laevigatus (Fieber). IOBC/WPRS Bulletin 19: 15-18.

Bueno, V. H. P. 2000. Controle Biológico de pragas: produção massal e controle de qualidade. Lavras: UFLA, 207 p.

Bueno, V. H. P.; S. M. Mendes \& L. M. Carvalho. 2006. Evaluation of a rearing-method for the predator Orius insidiosus. Bulletin of Insectology 59: $1-6$.

Bueno, V. H. P.; L. M. Carvalho \& N. Moura. 2007. Optimization of massrearing of Orius insidiosus: how far are we? Global IOBC Bulletin 3: $18-19$.

Carmona, R. \& H. D. C. Villas Boas. 2001. Dinâmica de sementes de Bidens pilosa no solo. Pesquisa Agropecuária Brasileira 36: 457-463.

Carvalho, L. M.; V. H. P. Bueno \& S. M. Mendes. 2005. Response of two Orius species to temperature. IOBC/ WPRS Bulletin 28: 43-46.

Castañé C. \& F. Zalom. 1994. Artificial oviposition substrate for rearing Orius insidiosus (Hemiptera: Anthocoridae). Biological Control 4: 88-91.

Coll, M. 1998. Living and feeding on plants in predatory heteroptera. p. 89-129. In: M. Coll \& J. R. Ruberson (eds.). Predatory Heteroptera: their ecology and use in biological control. Entomological Society of America, $233 \mathrm{p}$.

Diniz, A. J. F.; V. H. P. Bueno; A. R. Carvalho \& L. M. Carvalho. 2006. Desinfection of oviposition substrate with sodium hipochlorite: effects on some biological traits of Orius thyestes. IOBC/WPRS Bulletin 29: 215-218.

Filgueira, F. A. R. 2000. Novo manual de olericultura: agrotecnologia moderna na produção e comercialização de hortaliças. Viçosa-MG, Ed. Viçosa. 402 p.

Honda, J. Y.; Y. Nakashima \& Y. Hirose. 1998. Development, reproduction and longevity of Orius minutus and Orius sauteri (Heteroptera: Anthocoridae) when reared on Ephestia kuehniella eggs. Applied
Entomology and Zoology 33: 449-453.

Isenhour. D. J. \& K. V. Yeargan. 1981. Effect of temperature on the development of Orius insidiosus with notes on laboratory rearing. Annals of the Entomological Society of America 74: 114-116.

Lundgren, J. G. \& J. K. Fergen. 2006. The oviposition behavior of the predator Orius insidiosus: acceptability and preference for different plants. BioControl 51: 217-227.

Mendes, S. M.; V. H. P. Bueno \& L. M. Carvalho. 2005. Adequabilidade de diferentes substratos à oviposição do predador Orius insidiosus (Say) (Hemiptera: Anthocoridae). Neotropical Entomology 34: 415-421.

Murai, T.; Y. Narai \& N. Sugiura. 2001. Utilization of germinated broad bean seeds as an oviposition substrate in mass rearing of the predatory bug, Orius sauteri (Poppius) (Hemiptera: Anthocoridae). Applied Entomology and Zoology 36: 489-494.

Nagai, K. \& E. Yano. 1999. Effects of temperature on the development and reproduction of Orius sauteri (Poppius) (Heteroptera: Anthocoridae), a predator of Thrips palmi Karny (Thysanoptera: Thripidae). Applied Entomology and Zoology 34: 223-229.

Pereira, J. E. S. \& G. R. L. Fortes. 2004. Produção de mudas pré-básicas de batata por estaquia a partir de plantas micropropagadas. Horticultura Brasileira 22: 186-192.

Richards, P. C. \& J. M. Schmidt. 1996. The suitability of some natural and artificial substrates as ovipositional sites for the insidiosus flower bug, Orius insidiosus. Entomologia Experimentalis et Applicata 80: 325333.

Ruberson, J. R.; Y. J. Shen \& T. J. Kring. 2000. Photoperiod sensitivity and diapause in predator Orius insidious (Heteroptera: Anthocoridae). Annals of the Entomological Society of America 93: 1123-1130.

Silva, J. R. V.; N. V. Costa; O. S. Morais; M. A. Terra; S. R. Marchi \& E. O. Ono. 2004. Brotações de mini-tubérculos de sete cultivares de batata em função da concentração de bissulfureto de carbono. Horticultura Brasileira 22: 677-680.

Tommasini, M. G.; J. C. Van Lenteren \& G. Burgio. 2004. Biological traits and predation capacity of four Orius species on two prey species. Bulletin of Insectology 57: 79-93.

van den Meiracker, R. A. F. 1999. Biocontrol of western flower thrips by heteropteran bugs. Amsterdan, Thesis, $145 \mathrm{p}$.

van den Meiracker, R. A. F \& C. Sabelis. 1993. Oviposition sites of Orius insidiosus in sweet pepper. IOBC/WPRS Bulletin 6: 109-112.

Yano, E.; K. Nagai; K. Watanabe \& K. Yara. 2002. Biological parameters of Orius spp. for control of thrips in Japan. IOBC/ WPRS Bulletin 25: 305-308.

Wang, C. L.; P. C. Lee \& Y. J. Wu. 1999. Field augmentation of Orius strigicollis (Heteroptera: Anthocoridae) for the control of thrips in Taiwan. Disponível em: <http//:www.agnet.org/library/data/eb/eb500/ eb500.pdf.> Acesso: 25 out. 2003. 